\title{
The effect of external magnetic field on the density distributions and electromagnetic fields in the interaction of high-intensity short laser pulse with collisionless underdense plasma
}

\author{
Masoomeh Mahmoodi-Darian ${ }^{1} \cdot$ Mehdi Ettehadi-Abari $^{2} \cdot$ Mahsa Sedaghat $^{2}$
}

Received: 4 March 2015 / Accepted: 6 October 2015/Published online: 18 November 2015

(C) The Author(s) 2015. This article is published with open access at Springerlink.com

\begin{abstract}
Laser absorption in the interaction between ultra-intense femtosecond laser and solid density plasma is studied theoretically here in the intensity range $I \lambda^{2} \simeq 10^{14}-10^{16} \mathrm{~W} \mathrm{~cm}^{-2} \mu \mathrm{m}^{2}$. The collisionless effect is found to be significant when the incident laser intensity is less than $10^{16} \mathrm{~W} \mathrm{~cm}^{-2} \mu \mathrm{m}^{2}$. In the current work, the propagation of a high-frequency electromagnetic wave, for underdense collisionless plasma in the presence of an external magnetic field is investigated. When a constant magnetic field parallel to the laser pulse propagation direction is applied, the electrons rotate along the magnetic field lines and generate the electromagnetic part in the wake with a nonzero group velocity. Here, by considering the ponderomotive force in attendance of the external magnetic field and assuming the isothermal collisionless plasma, the nonlinear permittivity of the plasma medium is obtained and the equation of electromagnetic wave propagation in plasma is solved. Here, by considering the effect of the ponderomotive force in isothermal collisionless magnetized plasma, it is shown that by increasing the laser pulse intensity, the electrons density profile leads to steepening and the electron bunches of plasma become narrower. Moreover, it is found that the wavelength of electric and magnetic field oscillations increases by increasing the external magnetic field and the density distribution of electrons also grows in comparison to the unmagnetized collisionless plasma.
\end{abstract}

Masoomeh Mahmoodi-Darian

m.darian@kiau.ac.ir

1 Department of Physics, Karaj Branch, Islamic Azad University, Karaj, Iran

2 Physics Department and Laser Research Institute of Beheshti University, G.C., Evin, 19839 Tehran, Iran
Keywords Laser plasma interaction - Ponderomotive force - Underdense magnetized plasma - Nonrelativistic regime $\cdot$ Electrons density distribution

\section{Introduction}

Theoretical and experimental studies of electromagnetic wave propagation in magnetized plasmas are of key importance for a vast range of problems in space and laboratory physics [1-3]. The acceleration of electrons in the interaction of a high-intensity laser beam with plasma may have important applications in various domains such as laser particle acceleration, ion acceleration for fusion action, and the generation of intense and short-duration $\gamma$ ray sources for radiography [4-6]. Furthermore, the interaction of an ultra short high-intensity laser pulse with plasma without external dc magnetic field has been studied extensively [7-11]. When an external magnetic field is applied to the plasma, it is a medium capable to convert different initial energies to tunable coherent radiations. In 1976 it was shown that a linearly polarized laser beam interacting with hot magnetized plasma produces a radiative force along the electric field of laser beam. This magnetic radiation force at $5 \mathrm{MG}$ is as large as the ponderomotive force for a $3-\mu \mathrm{m}$ scale height [12]. In 2002, the generation of the huge azimuthal self-magnetic field of over 340 MG near the critical density surface was measured [13]. Two years later, this amount was scaled up to more than 700 MG by the same group in over dense plasma [14] while in underdense plasma fields in the order of 100 MG is presented [15]. The self-magnetic field in plasma is a function of laser intensity and Qiao et al. [16] showed, by their analytical model for Nd-glass laser at the intensity of $10^{20} \mathrm{~W} \mathrm{~cm}^{-2}$, the obtained magnetic field of about $90 \mathrm{MG}$. 
In studying the plasma parameters in an external magnetic field, the intensity should be in the same order where few megagauss magnetic field facilities for magnetized laserplasma experiments are established [17, 18]. A method is also described for choosing experimental parameters in studies of high energy density physics relevant to fusion energy, as well as other applications by using megagauss magnetic fields [19]. The structure of these systems is based on the compact megagauss magnetic field generation in single-turn coils which created new frontiers for scientific experiments [20].

Extremely high azimuthal magnetic fields play an essential role in the particle transport, propagation of laser pulses, laser beam self-focusing, penetration of laser radiation into the overdense plasma, and the plasma electron and ion acceleration, where the first direct measurements of high-energy proton generation (up to $18 \mathrm{MeV}$ ) and propagation into a solid target during such intense laser plasma interactions were reported. Measurements of the deflection of these energetic protons were carried out which imply that magnetic fields in excess of $30 \mathrm{MG}$ exist inside the target [21]. Although some valuable experimental works have been reported, the introduced analytical works did not properly explain the fundamental parameters of the magnetized collisionless plasma, including electric field oscillation, electron density distribution or the derived equations, being related to very special conditions [22, 23]. In the recent studying of plasma, such as ponderomotive acceleration of plasma in the applied magnetic field and improving of self-focusing in presence of the applied external magnetic fields, the range of magnetic field intensities are in the range of tens of megagauss [24, 25]. Based on the report of Gupta et al. [25], they did not have the effect of external magnetic field up to $20 \mathrm{MG}$ in an underdense subrelativistic plasma and the effect is enhanced by increasing the magnetic field intensity up to $45 \mathrm{MG}$, where we have noticed an almost similar dependence of mentioned parameters to the magnetic field intensity in the present analytical work. Although some valuable experimental works are reported, the introduced analytical works did not properly explain the fundamental parameters of the magnetized plasma including electric and magnetic field oscillations or the derived equations being related to very special conditions [26-32].

Furthermore, the numerical analysis of high-power laser propagation in a magnetized plasma was considered by Druce et al. [33]. Here, a $\mathrm{CO}_{2}$ laser with $10.6 \mu \mathrm{m}$ wavelength interacts with magnetically confined plasma in the density range of $10^{17}-10^{18} \mathrm{~cm}^{-3}$. Here, by using of the momentum and energy equations for the plasma species and assuming the dependence of $T_{e}$ to the coordinates, the laser propagation in magnetized confined collisionless plasma is analyzed. In addition, in the previous work [34], we studied the nonlinear propagation of an intense laser pulse through an underdense magnetized plasma. Here, we considered the semi-infinite plasma in the region of $z \phi 0$ in attendance of a constant external magnetic field $B_{0}$ in $y$ direction. The laser pulse is irradiated perpendicular into the plasma in $z$ direction and the polarization of the incident wave is assumed to be linear. In this work it is obvious that the high azimuthally external magnetic field is considered [i.e., $\mathbf{k}=(0,0, k), \mathbf{B}_{\mathbf{0}}=\left(0, B_{0}, 0\right), \mathbf{E}=\left(E_{x}, 0,0\right)$, $\left.\mathbf{B}=\left(0, B_{y}, 0\right)\right]$.

In the current theoretical study, by considering the circular symmetry case where the electric and magnetic fields of the laser are perpendicular to $B_{0}$ (external magnetic field) and with assumption an azimuthally polarized laser pulse $\left(E_{\theta}(z, t)=\hat{\theta} E_{\theta}(z) \exp (-i \omega t)\right)$, where $\hat{\theta}$ is the unit vector in the azimuthal direction, we have studied the nonlinear structure of electromagnetic wave propagation of an ultra-short laser beam in underdense collisionless magnetized plasma. An external magnetic field is applied in the direction of laser beam propagation in homogeneous plasma. To achieve the nonlinear equation for the electric field in the plasma, we use the Maxwell equations and the equation of electrons motion while taking into account the average ponderomotive force per unit volume acting on the plasma electrons. Since the ion mass is much greater than the electron mass, we neglect the effect of the ponderomotive force on the ions. In order to imply the magnetic field in our formulation, we use the external plasma current density $\mathbf{J}=-4 \pi n_{e} e \mathbf{V}$ in Ampere's law, in which $e, n_{e}$, and $\mathbf{V}$ are the electron charge, the electron density and the drift velocity of electrons. Furthermore, using the Maxwell and the transfer momentum equations, we found the dispersion relation of the transverse wave, in isothermal collisionless magnetized plasma. In our work, we show that in the presence of external magnetic field in the direction of laser beam propagation, the density of the electrons increases.

This work is organized as follows: after introduction the theoretical model and formulation are presented in Sect. II. Here, the basic equations and fundamental assumptions are given. In Sect. III, we have discussed the numerical method used in this work, and finally Sect. IV is devoted to conclusion.

\section{The theoretical model and formulation}

In order to formulate the nonlinear propagation of an intense laser pulse through an underdense magnetized collisionless isothermal plasma in nonrelativistic regime let us consider a semi-infinite plasma $(z>0)$ in a constant external magnetic filed $\mathbf{B}_{\mathbf{0}}$ in the $z$ direction. In this model, we assume circular symmetry and a field $\mathbf{E}$ that is 
azimuthally polarized $\left(E_{\theta}(z, t)=\hat{\theta} E_{\theta}(z) \exp (-i \omega t)\right)$ and it propagates in the $+z$ direction, where $\hat{\theta}$ is the unit vector in the azimuthal direction. In this case we should mention that polarization is one of the most important characteristics of laser radiation. While determining the polarization state of the beam, one can speak about type of polarization at the point of the beam cross section, homogeneity of ellipsometrical parameters over the beam cross section, and stability of polarization characteristics in time. From the nowadays viewpoint, the conventional types of polarization have substantial disadvantages. In the case of linear polarization, the parameters of the beam interaction with the matter depend upon the direction of polarization. In the case of circular polarization, these parameters are time averaged, i.e., not optimum from view point either of minimum losses or maximum absorption. The modes with inhomogeneous polarization, radial or azimuthal, are known in the laser resonator theory. In the case of radial (azimuthal) polarization, the direction of the electrical vector in the plane of the beam cross section is parallel (perpendicular) to the radial direction. Here, we consider a semi-infinite plasma in $z>0$ region in a constant external magnetic filed $\mathbf{B}_{\mathbf{0}}$ in the $z$ direction. $z>0$ region is taken to be filled with a homogeneous density profile of plasma with plasma-vacuum interface at $z=0$. It should be noted that, in the problem of interaction of laser beams with plasma, if the vector $\mathbf{E}$ is parallel to the plane of incidence (radial polarization), the resonance absorption is maximum and if the vector $\mathbf{E}$ is perpendicular to the plane of incidence (azimuthal polarization), no resonance absorption occurs. In this case the electrons in plasma oscillate in the wave electrical field along lines of equal density without generating electrostatic fields. Such type of polarization that we used in this manuscript is useful for investigating ponderomotive forces affecting electron density profile [35-37]. As we see from Fig. 1, the electromagnetic wave enters normally into the plasma slab.

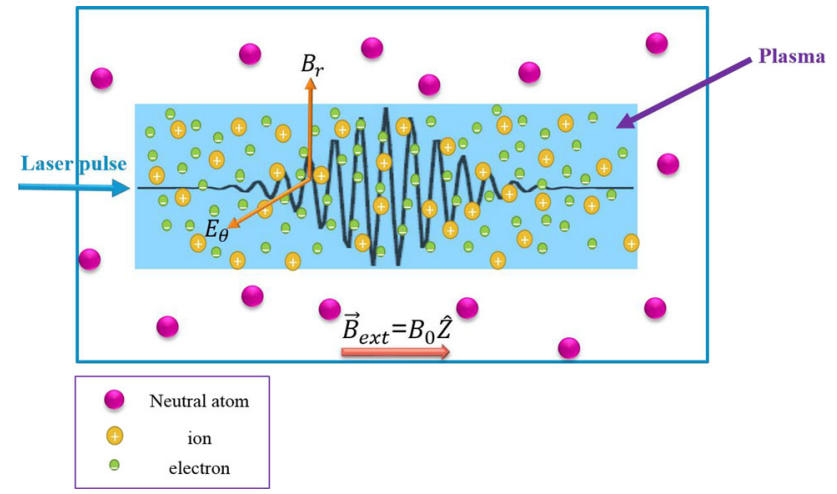

Fig. 1 Schematic view of the laser pulse propagation through the magnetized underdense plasma is shown. Direction of the applied uniform magnetic field $B_{0}$ is in $z$ direction
To develop the wave equation for the oscillating electric and magnetic fields, one can start with Faraday's induction and Ampere's laws. By considering these Maxwell's equation, we have:

$\nabla \times \mathbf{E}=-i \omega \mathbf{B}$

$\nabla \times \mathbf{B}=\mu_{0}\left(\mathbf{J}-i \varepsilon_{0} \omega \mathbf{E}\right)$.

Now, if we assume that the laser electromagnetic transverse wave fields with frequency $\omega$ propagate through plasma along the $z$ direction, the laser pulse electric field evolves as

$\frac{\mathrm{d}^{2}}{\mathrm{~d} z^{2}} E_{\theta}+k_{0}^{2} E_{\theta}+i \omega \mu_{0} J_{\theta}=0$.

Here, we consider the parameter $\mu_{0} \varepsilon_{0} \omega^{2}=k_{0}^{2}=\omega^{2} / c^{2}$ as the vacuum propagation constant. Now, to obtain the laser pulse electric field in plasma, we should use the appropriate electron current density $J_{\theta}$ in Eq. (3). As we know, the plasma electron current density equation is $J_{\theta}=-n_{e} e V_{e \theta}$ and for this purpose we should consider the equation of electrons motion in collisionless isothermal plasma as

$m_{e} n_{e} \frac{\mathrm{d} \mathbf{V}_{\mathbf{e}}}{\mathrm{d} t}=-\mathrm{e} n_{e}\left(\mathbf{E}+\frac{1}{c} \mathbf{V}_{\mathbf{e}} \times \mathbf{B}\right)-\nabla P_{e}+\mathbf{F}_{\mathbf{P e}}$,

where $m_{e}, V_{e}, P_{e}=n_{e} T_{e}$ and $\mathbf{F}_{\mathbf{P e}}$ are electron mass, electron velocity, pressure of electrons, and the average ponderomotive force defined by the laser pulse envelope. In this work, since the ion mass is much greater than the electron mass and $\omega \gg \omega_{p i}$, where $\omega_{p i}=\sqrt{\frac{4 \pi n_{i} e^{2}}{m_{i}}}$, we neglect the ion motions. Then by writing Eq. (4) in the $\hat{r}$ and $\hat{\theta}$ directions, the $v_{r}$ and $v_{\theta}$ components of electron velocity of magnetized collisionless isothermal plasma can be written as

$v_{r}=\frac{i e}{m_{e} \omega} \frac{E_{r}-i\left(\frac{\omega_{c e}}{\omega}\right) E_{\theta}}{1-\left(\frac{\omega_{c e}}{\omega}\right)^{2}}$,
$v_{\theta}=\frac{-i e}{m_{e} \omega} \frac{E_{\theta}-i\left(\frac{\omega_{c e}}{\omega}\right) E_{r}}{1-\left(\frac{\omega_{c e}}{\omega}\right)^{2}}$.

Here $E_{r}$ and $E_{\theta}$ are radial and azimuthal electric field components, respectively. Now by considering an azimuthally polarized laser beam $\left(E_{r}=0\right)$, we reach to the following equations for $v_{r}$ and $v_{\theta}$ components:

$$
\begin{aligned}
& v_{r}=\frac{e}{m_{e} \omega}\left(\frac{\omega_{c e}}{\omega}\right) \frac{E_{\theta}}{1-\left(\frac{\omega_{c e}}{\omega}\right)^{2}}, \\
& v_{\theta}=\frac{-i e}{m_{e} \omega} \frac{E_{\theta}}{1-\left(\frac{\omega_{c e}}{\omega}\right)^{2}} .
\end{aligned}
$$

In the above equations $\omega_{c e}=e B_{0} / m_{e} c$ is the electrons cyclotron frequency due to the existence of the external magnetic field and $\omega$ is the laser pulse frequency. Here, the plasma electrons current density is obtained as 
$J_{\theta}=n_{e} e V_{e \theta}=\frac{i \varepsilon_{0} \omega_{p e}^{2} E_{\theta}}{\omega\left(1-\frac{\omega_{c e}^{2}}{\omega^{2}}\right)}$,

where $\omega_{p e 0}=\sqrt{\frac{4 \pi n_{e} e^{2}}{m_{e}}}$ is the plasma electron frequency and $n^{e}$ is the plasma density. Now, we consider the homogeneous collisionless magnetized isothermal plasma in the presence of the ponderomotive force due to the laser pulse. In this case, in the steady state, the ponderomotive force in attendance of the external magnetic field can be balanced with the electron pressure gradient force. Consequently, according to the momentum transfer Eq. (4) in the laser pulse propagation direction and assuming that the electron temperature $T_{e}$ is independent of coordinates, we have

$\frac{-n_{e} e^{2}}{2 m_{e}\left(\omega^{2}-\omega_{c e}^{2}\right)} \frac{\mathrm{d} E_{\theta}^{2}}{\mathrm{~d} z}=T_{e} \frac{\mathrm{d} n_{e}}{\mathrm{~d} z}$,

where $T_{e}$ is given in energy unit. Integrating Eq. (10) from $n_{e 0}$ to $n_{e}$, the electrons density becomes a function of laser pulse intensity as

$n_{e}(z)=n_{e 0} \exp \left(-\frac{e^{2} E_{\theta}^{2}(z)}{m_{e} T_{e}\left(\omega^{2}-\omega_{c e}^{2}\right)}\right)$.

It should be noted that, in the intermediate intensities $10^{14}-10^{16} \mathrm{~W} \mathrm{~cm}^{-2} \mu \mathrm{m}^{2}$ with laser pulse duration of order a few ns, it is convenient to assume that $T_{e}$ is constant and the dominant spatial dependence comes from the electron density $\left(n_{e}\right)$. This equation shows that the electron density is modified by the pondermotive force. Furthermore, by substituting Eq. (11) into Eq. (9) and using Eq. (3), the nonlinear equation for electric field propagation in collisionless plasma is obtained as

$$
\begin{aligned}
\frac{\mathrm{d}^{2}}{\mathrm{~d} z^{2}} E_{\theta} & +\left(\frac{\omega^{2}}{c^{2}}\right)\left\{1-\left(\frac{\omega_{p e 0}^{2}}{\omega^{2}\left(1-\frac{\omega_{c e}^{2}}{\omega^{2}}\right)}\right)\right. \\
& \left.\times \exp \left(-\frac{e^{2} E_{\theta}^{2}}{m_{e} T_{e}\left(\omega^{2}-\omega_{\mathrm{ce}}^{2}\right)}\right)\right\} E_{\theta}=0 .
\end{aligned}
$$

In addition, the dielectric constant of a magnetized collisionless isothermal plasma can be found as follows:

$\varepsilon=1-\frac{\omega_{\mathrm{pe} 0}^{2} \exp \left(-\frac{e^{2} E_{\theta}^{2}}{m_{e} T_{e}\left(\omega^{2}-\omega_{\mathrm{ce}}^{2}\right)}\right)}{\left(\omega^{2}-\omega_{\mathrm{ce}}^{2}\right)}$.

As we see, it should be noted that the electromagnetic wave equation coupled with the equations of momentum transfer, particle conservation and energy in their stationary form and they are solved for obtaining the dielectric permittivity. Now, with having the dielectric permittivity variations, the electric field propagation through the plasma along the $\mathrm{Z}$ direction is obtained as Eq. (3). In addition, with having the electric field variation according to the Eq. (3), we can reach the quiver velocities of electrons Eqs. (5) and (6) and their variations in plasma. Here, the velocities depend on the electrons density, temperature parameters. In other words, as $E_{\theta}$ was obtained from Eq. (3), in which the current density $J_{\theta}$ depends on plasma electrons' density and temperature, we can conclude that the theta component of electric field is a function of temperature. Furthermore, as we know, for obtaining the ponderomotive force we should use the nonlinear theory (Ref. [22]). Here, it should be mentioned that the following equations, i.e., momentum transfer and the laser pulse electric field propagation in plasma have the directional dependence on the nonlinear ponderomotive force, and according to it, we conclude that all of them are nonlinear. Now, we analyze the nonlinear wave equation, dielectric permittivity, and the plasma electron density distribution in such plasmas. Substituting Eq. (11) into Eqs. (12) and (13), results in the inhomogeneous dielectric permittivity and the nonlinear equation for the electric field propagation in collisionless isothermal magnetized plasma: $\varepsilon=1-\left(\frac{\omega_{p e 0}^{2}}{\left(\omega^{2}-\omega_{c e}^{2}\right)}\right) \times \exp \left(-\frac{e^{2} E_{\theta}^{2}(z)}{m_{e} T_{e}\left(\omega^{2}-\omega_{c e}^{2}\right)}\right)$,

$$
\begin{aligned}
\frac{\mathrm{d}^{2}}{\mathrm{~d} z^{2}} E_{\theta} & +\left(\frac{\omega^{2}}{c^{2}}\right)\left\{1-\left(\frac{\omega_{\mathrm{pe} 0}^{2}}{\left(\omega^{2}-\omega_{\mathrm{ce}}^{2}\right)}\right)\right. \\
& \left.\times \exp \left(-\frac{e^{2} E_{\theta}^{2}(z)}{m_{e} T_{e}\left(\omega^{2}-\omega_{\mathrm{ce}}^{2}\right)}\right)\right\} E_{\theta}=0 .
\end{aligned}
$$

\section{Results and discussion}

In sect. II, we investigated the theoretical model and formulated the nonlinear ponderomotive force effects on isothermal collisionless magnetized underdense plasma. From Eq. (15), it is clear that this equation is intensively nonlinear and does not have any analytical solution. Thus, we use the fourth order Runge-Kutta method to solve this equation numerically and find the mentioned changes inside plasma. In order to obtain the laser pulse electric field, and the electron density profiles, we introduce some dimensionless variables as follows:

$a=\frac{e E_{\theta}}{m_{e} c_{s} \omega}, \Omega_{\mathrm{pe}}=\frac{\omega_{\mathrm{pe} 0}}{\omega}$,

$\xi \frac{z \omega}{c}, \omega_{c e 0} \frac{\omega_{c e}}{\omega}$,

where $c_{s}=\left(\frac{T_{e}}{m_{e}}\right)^{1 / 2}$ is the sound velocity. Using these dimensionless parameters, Eqs. (11), (14), and (15) are written as follows: 
$\frac{n_{e}}{n_{e 0}}=\exp \left(\frac{-a^{2}}{1-\omega_{c e 0}^{2}}\right)$,

$\varepsilon=1-\frac{\Omega_{p e}^{2}}{1-\omega_{c e 0}^{2}} \exp \left(\frac{-a^{2}}{1-\omega_{c e 0}^{2}}\right)$,

$\frac{d^{2} a}{d \xi^{2}}+\left\{1-\left(\frac{\Omega_{p e}^{2}}{1-\omega_{c e 0}^{2}}\right) \times \exp \left(\frac{-a^{2}}{1-\omega_{c e 0}^{2}}\right)\right\} a=0$.

Here, in the case of collisionless magnetized isothermal plasmas the following parameters of the laser beam and plasma have been chosen: $\frac{\omega_{p e 0}}{\omega}=0.6$ and $T_{e 0}=1 \mathrm{KeV}$. As we know, in plasma when the condition of $\left(n_{e}<n_{\text {critical }}\right)$ is satisfied the plasma is underdense and in the other case when this condition is not satisfied the plasma is overdense. Here in this work, because of the parameter $\frac{n_{e}}{n_{\text {critical }}}$ is proportional with $\omega_{p e}^{2} / \omega^{2}\left(\frac{n_{e}}{n_{\text {critical }}}=\frac{\omega_{p e}^{2}}{\omega^{2}}\right)$, the range of $\frac{n_{e}}{n_{\text {critical }}}$ is 0.36 and $n_{e}$ is very small in comparison with $n_{\text {critical }}$ and the plasma is underdense. In Fig. 2a, b, the effect of increasing laser intensity on the propagation of the electric field and the distribution of the electron density of magnetized collisionless plasma of this medium are shown.

In these figures the normalized external magnetic field is taken as $\omega_{c e} / \omega=0.4$. Since laser intensity is proportional to the square of amplitude, the amplitude of the electric field in plasma is increased with increasing laser intensity. Furthermore, due to further decrease in electron density in the higher laser intensities, more decrease in the wavelength of fields takes place. The steepening of the electron density distribution is enhanced with an increase in laser pulse intensity. It is seen that when laser intensity is increased, the oscillations of the electron density become highly peaked and at the same time, their wavelengths tend to decrease. The physical reason of this effect is that since $\lambda$ (the oscillation wavelength) is proportional to $\varepsilon^{-1 / 2}$, by increasing the dielectric constant the wavelength of electron's oscillation is decreased. It is noticeable that by increasing laser pulse intensity, the oscillations of the effective permittivity become highly peaked and the wavelength of these oscillations is decreased. Furthermore, we can see that by increasing laser intensity the oscillations of the effective permittivity are deviated from sinusoidal shape more intensively. Figure $3 \mathrm{a}, \mathrm{b}$ shows the effect of the magnitude increment of the external magnetic field on the profiles of the electric field and the electron density distribution of this medium in underdense collisionless isothermal magnetized plasma in the situation of constant laser intensity. In the presence of the external magnetic field parallel to the laser pulse propagation direction, the electrons density distribution is increased in comparison to unmagnetized collisionless isothermal plasma. In this case the electron density distribution is increased more in
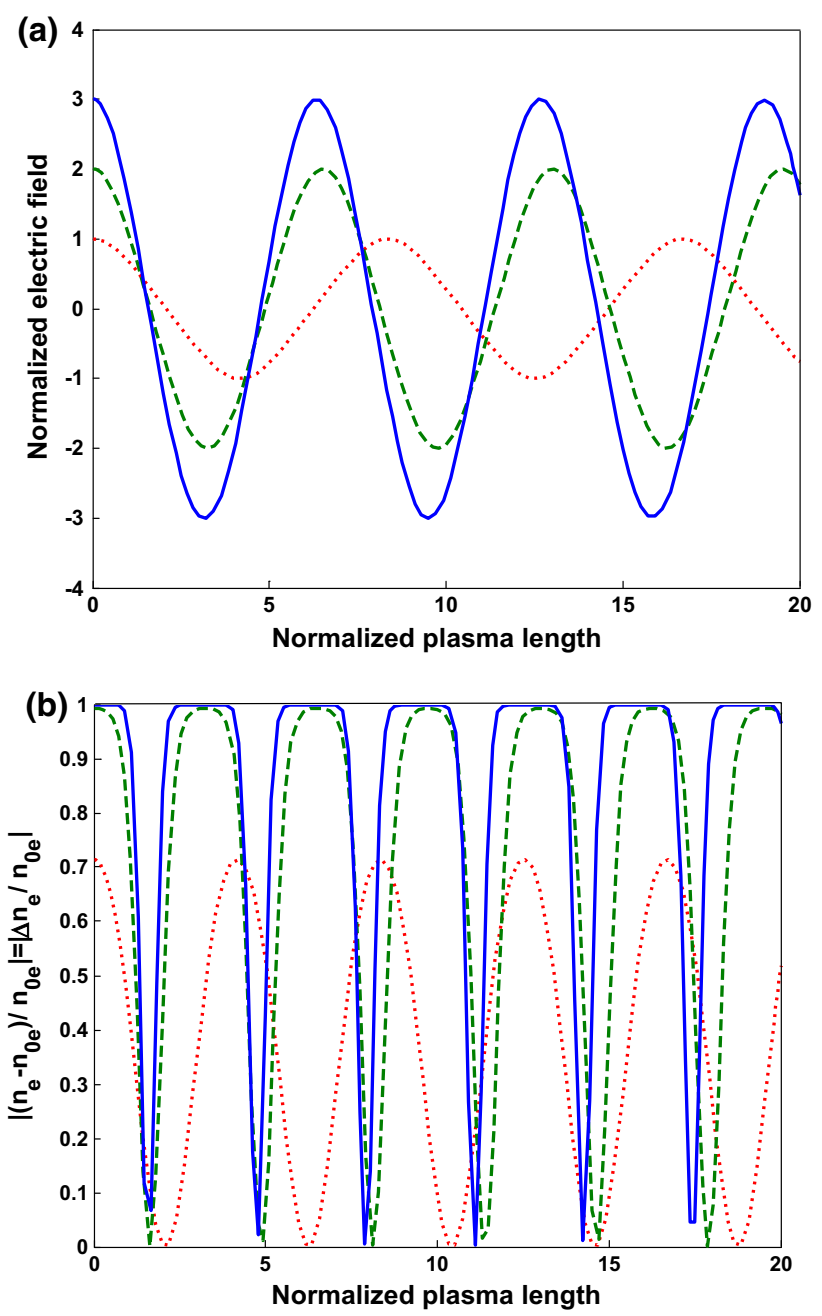

Fig. 2 The effect of increasing the laser pulse intensity in the case of the collisionless magnetized and isothermal plasma on the variations of the a normalized electric field, $\mathbf{b}$ normalized electrons density $\Delta n_{e} / n_{e 0}$. The dimensionless laser pulse intensities are $a_{0}=1$ (dotted line), $a_{0}=2$ (dotted-dashed line), and $a_{0}=3$ (solid line). Electron temperature is $T_{e}=1 \mathrm{keV}$, the normalized cyclotron frequency is $\frac{\omega_{c e}}{\omega}=0.4$, and the normalized plasma frequency is taken as $\frac{\omega_{p e 0}}{\omega}=0.6$

comparison with the electron density profile presented in the Ref. [34]. Here, the laser pulse should transfer more energy to the plasma electrons compared to unmagnetized plasma. It leads to an increase in the wavelength of the electric field. It is obvious that by increasing the external magnetic field, as a result of the increase of the electrons density distribution, the dielectric permittivity constant is decreased.

\section{Summary and conclusion}

In this paper, we have formulated the nonlinear propagation of an intense laser pulse through underdense magnetized collisionless isothermal plasma. From the results, one can see, in magnetized plasma, field wavelength is 

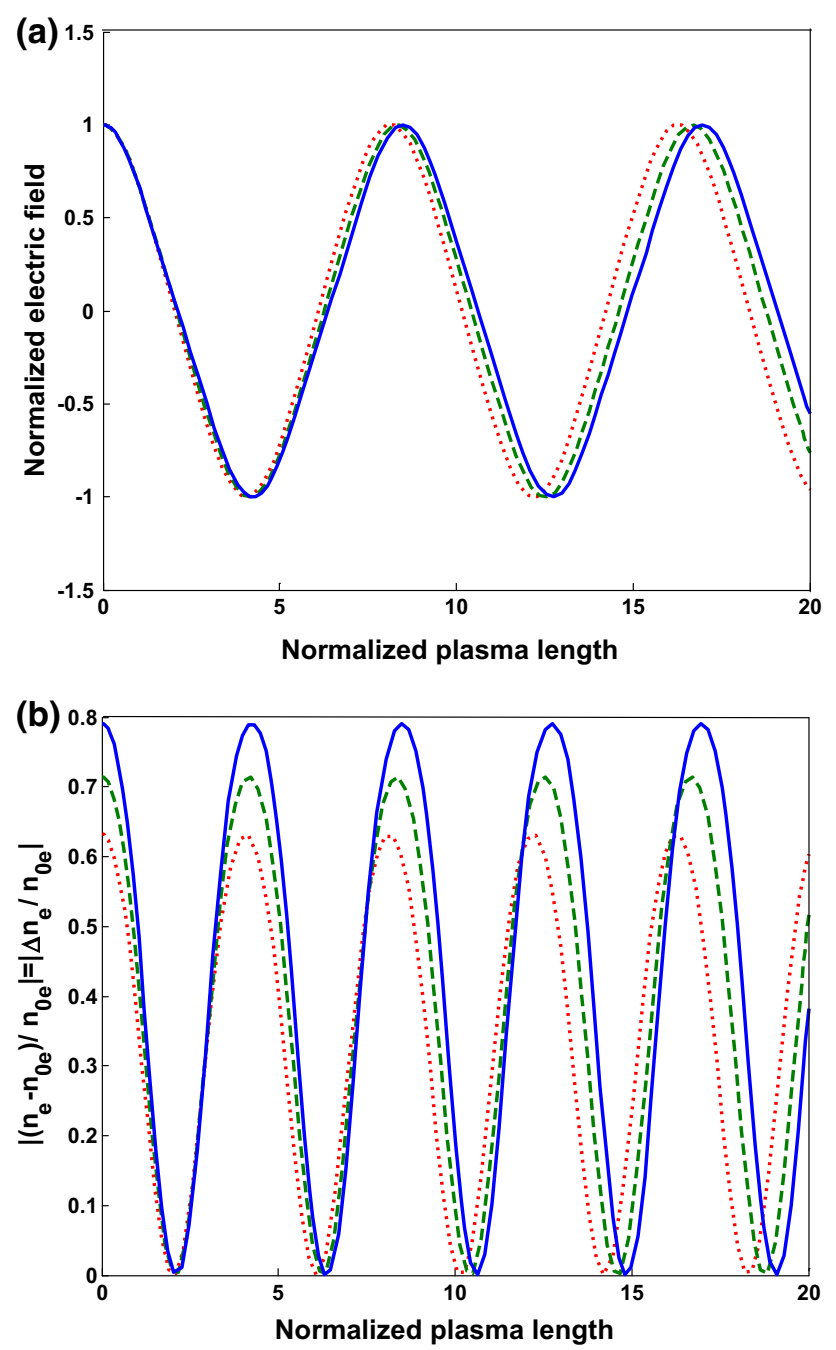

Fig. 3 The effect of increasing the external magnetic field in the case of the collisionless magnetized and isothermal plasma at the dimensionless laser pulse intensity of $a_{0}=1$ on the variations of a: normalized electric field, and $\mathbf{b}$ normalized electron density, $\Delta n_{e} / n_{e 0}$ for different values of normalized cyclotron frequency frac $\omega_{c e} \omega=0$ (dotted line), $\frac{\omega_{c e}}{\omega}=0.4$ (dotted-dashed line), $\frac{\omega_{c e}}{\omega}=0.6$ (solid line) is shown. Electron temperature is $T_{e}=1 \mathrm{keV}$, and the normalized plasma frequency is taken as $\frac{\omega_{p e 0}}{\omega}=0.6$

increased relative to the wavelength in unmagnetized plasma in the similar conditions. Also in the presence of the external magnetic field, the plasma electron density is increased and due to this effect, the effective permittivity of the mentioned plasma is decreased. Our numerical results are in good agreement with the mentioned work ( $\mathrm{R}$. L. Druce). Magnetized plasma plays as a capable medium to convert different inertial energies to tunable coherent radiation. This is because the electron density distribution is proportional to the plasma frequency and the magnitude of the external magnetic field at constant laser intensity. By adjusting the magnitude of the external magnetic field, the desired value of the electron density can be produced.
Acknowledgments This work was supported by Islamic Azad University, Karaj Branch. This work is a part of the project "The interaction of high intensity short laser pulse with the cold and magnetized plasma".

Open Access This article is distributed under the terms of the Creative Commons Attribution 4.0 International License (http://crea tivecommons.org/licenses/by/4.0/), which permits unrestricted use, distribution, and reproduction in any medium, provided you give appropriate credit to the original author(s) and the source, provide a link to the Creative Commons license, and indicate if changes were made.

\section{References}

1. Ginzburg, V.L.: The Propagation of Electromagnetic Waves in Plasmas. Pergamon, Oxford (1970)

2. Chen, F.F.: Introduction to Plasma Physics and Controlled Fusion. Springer, Berlin (2010)

3. Mourou, G.A., Tajima, T., Bulanov, S.V.: Rev. Mod. Phys. 78, 309 (2006)

4. Esarey, E., Goloviznin, R., Trans, I.E.E.E.: Plasma Sci. 24, 252 (1996)

5. Cowan, T.E., Hunt, A.W., Phillips, T.W., Wilks, S.C., Perry, M.D., Brown, C., Fountain, W., Hatchett, S., Johnson, J., Key, M.H., Parnell, T., Pennington, D.M., Snavely, R.A., Takahashi, Y.: Rev. Sci. Instrum. 70, 265 (1999)

6. Mangles, S.P.D., Thomas, A.G.R., Lundh, O., Landau, F., Kaluza, M.C., Persson, A., Wahistrom, C.G., Krusheinick, K., Najmudin, Z.: Phys. Plasmas 14, 056702 (2007)

7. Gordienko, S., Pukhov, A.: Phys. Plasmas 12, 043109 (2005)

8. Shokri, B., Niknam, A.R.: Phys. Plasmas 13, 113110 (2006)

9. Davis, J., Petrov, G.M., Velikovich, A.L.: Phys. Plasmas 12, $123102(2009)$

10. Wei, M.S., Mangles, S.P.D., Najmudin, Z., Walton, B., Gopal, A., Tatarakis, M., Krushelnic, K.: Phys. Phys. Rev. Lett. 93, 155003 (2004)

11. Korovin, S.D., Kurkan, I.K., Loginov, S.V., Pegal, I.V., Polevin, S., Volkov, S.N., Zherlitsyn, A.: Laser Part. Beams 21, 175 (2003)

12. Stamper, J.A., Bonder, S.E.: Phys. Rev. Lett. 37, 435 (1976)

13. Tatarakis, M., Watts, I., Beg, F.N., Clark, E.L., Dangor, A.E., Gopal, A., Haines, M.G., Norreys, P.A., Wagner, U., Wei, M.-S., Zepf, M., Krushelnick, K.: Nature (London) 415, 280 (2002)

14. Wagner, U., Tatarakis, M., Gopal, A., Beg, F.N., Clark, E.L., Dangor, A.E., Evanns, R.G., Haines, M.G., Norreys, P.A., Wei, M.S., Zeft, M., Krushelnick, K.: Phys. Rev. Lett. 70, 026401 (2004)

15. Pukhov, A., meyer-ter-Vehn, J., Gopal, A.: Phys. Rev. Lett. 76, 3975 (1996)

16. Qiao, B., Zhu, S., Zheng, C.Y., He, X.T.: Phys. Plasmas 12, 053104 (2005)

17. Presura, R., Plechaty, C., Martinez, D., Bakeman, M.S., Laca, P.J., Haefner, C., Astanovit-skiy, A.L., Thompson, M., Trans, I.E.E.E.: Plasma Sci. 36, 17 (2008)

18. Struve, K.W., Porter, J.L., Rovang, D.C.: Megagauss field generation for highenergy-density plasma science experiments, SANDIA report no. SAND2008-7015, (2008)

19. Siemon, R.E., Bauer, B.S., Awe, T.J., Angelova, M.A., Fuelling, S., Goodrich, T., Lindemuth, I.R., Makhin, V., Atchison, W.L., Faehl, R.J., Reinovsky, R.E., Turchi, P.J., Degnan, J.H., Ruden, E.L., Frese, M.H., Garanin, S.F., Mokhov, V.N.: J. Fusion Energy 27, 235 (2008)

20. Portugall, O., Puhlmann, N., Mller, H.U., Barczewski, M., Stolpe, I., von Ortenberg, M.: J. Phys. D 32, 2354 (1999) 
21. Clark, E.L., et al.: Phys. Rev. Lett. 84, 6703 (2000)

22. Eliezer, S.: The interaction of high power lasers with plasmas, Chap. III. IOP, Bristol (2002)

23. Liu, S.C.S., Tripathi, V.K.: Interaction of electromagnetic waves with electron beams and plasmas, Chap. VI. World Scientific, Singapore (1994)

24. Sharma, A., Tripathi, V.K.: Phys. Plasmas 16, 043103 (2009)

25. Gupta, D.N., Hur, M.S., Suk, H.: Appl. Phys. Lett. 91, 081505 (2007)

26. Yugami, N., Higashiguchi, T., Gao, H., Sakaii, S., Takahashi, K., Ito, H., Nishida, Y., Katsouleas, T.: Phys. Rev. Lett. 89, 065003 (2002)

27. Dorranian, D., Starodubtsev, M., Kawakami, H., Ito, H., Yugami, N., Nishid, Y.: Phys. Rev. E 68, 026409 (2003)

28. Dorranian, D., Ghoranneviss, M., Starodubtsev, M., Ito, H., Yugami, N., Nishida, Y.: Phys. Lett. A 331, 77 (2004)
29. Tsung, F.S., Morales, G.J., Tonge, J.: Phys. Plasmas 14, 042101 (2007)

30. Norreys, P.A., Beg, F.N., Sentoku, Y., Silva, L.O., Smith, R.A., Trines, R.M.G.M.: Phys. Plasmas 16, 041002 (2009)

31. Davis, J., Petrov, G.M., Velikovich, A.L.: Phys. Plasmas 13, 041002 (2009)

32. Pathak, V.B., Tripathi, V.K.: Phys. Plasmas 14, 022105 (2007)

33. Druce, R.L., Kristiansen, M., Hagler, M.O.: Proceedings of the Indian Academy of Sciences - Section A 86, 255-263 (1977)

34. Sadighi-Bonabi, R., Etehadi-Abari, M.: Phys. Plasmas 17, 032101 (2010)

35. Nesterov, A.V., Nieziev, V.G.: J. Phys. D Appl. Phys. 33, 1817-1822 (2000)

36. Niziev, V.G., Nesterov, A.V.: J. Phys. D Appl. Phys. 32, 1455-1461 (1999)

37. Jordan, R.H., Hall, D.G.: Opt. Lett. 19, 427-429 (1994) 